\title{
Markov Logic: A Unifying Language for Structural and Statistical Pattern Recognition
}

\author{
Pedro Domingos, Stanley Kok, Daniel Lowd, Hoifung Poon, Matt Richardson, \\ Parag Singla, Marc Sumner, and Jue Wang
}

\begin{abstract}
Effective pattern recognition requires understanding both statistical and structural aspects of the input, but in the past these have mostly been handled separately. Markov logic is a powerful new language that seamlessy combines the two. Models in Markov logic are sets of weighted formulas in first-order logic, interpreted as templates for features of Markov random fields. Most statistical and structural models in wide use are simple special cases of Markov logic. Learning algorithms for Markov logic make use of conditional likelihood, convex optimization, and inductive logic programming. Inference algorithms combine ideas from Markov chain Monte Carlo and satisfiability testing. Markov logic has been successfully applied to problems in information extraction, robot mapping, social network modeling, and others, and is the basis of the open-source Alchemy system.
\end{abstract}

